\title{
LocExpress: a web server for efficiently estimating expression of novel transcripts
}

\author{
Mei Hou', Feng Tian ${ }^{1,2}$, Shuai Jiang ${ }^{1}$, Lei Kong ${ }^{1}$, Dechang Yang ${ }^{1}$ and Ge Gao ${ }^{\text {** }}$ \\ From 15th International Conference On Bioinformatics (INCOB 2016) \\ Queenstown, Singapore. 21-23 September 2016
}

\begin{abstract}
Background: The temporal and spatial-specific expression pattern of a transcript in multiple tissues and cell types can indicate key clues about its function. While several gene atlas available online as pre-computed databases for known gene models, it's still challenging to get expression profile for previously uncharacterized (i.e. novel) transcripts efficiently.

Results: Here we developed LocExpress, a web server for efficiently estimating expression of novel transcripts across multiple tissues and cell types in human (20 normal tissues/cells types and 14 cell lines) as well as in mouse (24 normal tissues/cell types and nine cell lines). As a wrapper to RNA-Seq quantification algorithm, LocExpress efficiently reduces the time cost by making abundance estimation calls increasingly within the minimum spanning bundle region of input transcripts. For a given novel gene model, such local context-oriented strategy allows LocExpress to estimate its FPKMs in hundreds of samples within minutes on a standard Linux box, making an online web server possible.
\end{abstract}

Conclusions: To the best of our knowledge, LocExpress is the only web server to provide nearly real-time expression estimation for novel transcripts in common tissues and cell types. The server is publicly available at http://loc-express. cbi.pku.edu.cn.

Keywords: Expression estimation, Transcriptome, RNA-Seq, Web server

\section{Background}

The rapid growth of high-throughput RNA-Seq data enables thousands of novel transcripts discovered annually, with the long noncoding RNAs (lncRNAs) as the major repertoire [1]. RNA expression profile provides important functional hints, which is particularly helpful for novel lncRNAs due to their largely elusive mechanisms [2]. While several expression atlas databases [3-5] work well for known gene models, they cannot handle novel transcripts which were not pre-calculated (also see Additional file 1: Table S1). On the other hand, the ab initio analysis of raw RNA-Seq data [6-8], which can survey the transcriptome global picture with both known and novel

\footnotetext{
* Correspondence: gaog@mail.cbi.pku.edu.cn

'State Key Laboratory of Protein and Plant Gene Research, College of Life

Sciences, Center for Bioinformatics, Peking University, Beijing 100871,

People's Republic of China

Full list of author information is available at the end of the article
}

gene models, is time consuming and hardly practical for bench biologists.

Here we propose LocExpress, a local context-oriented expression abundance estimation tool for novel transcripts. For a given gene model, LocExpress estimates its abundance only based on its minimum spanning bundle (MSB) region instead of reanalyzing the whole transcriptome. Such context-oriented strategy enables the nearly real-time expression profiling for a novel transcript in hundreds of samples, with the same accuracy of standard pipelines. To help bench biologists, we made LocExpress publicly available as a web server at http://loc-express.cbi.pku.edu.cn. Currently, the website supports instant abundance estimation across 101 human and mouse samples (Table 1 and 2, also see Additional file 2 for the full sample list). 
Table 1 LocExpress supports expression estimation in common tissues/cells

\begin{tabular}{|c|c|c|}
\hline & Human & Mouse \\
\hline Circulatory system & Heart, Whole blood & Heart \\
\hline Digestive system & $\begin{array}{l}\text { Colon, Liver, Pancreas, } \\
\text { Stomach }\end{array}$ & $\begin{array}{l}\text { Colon, Duodenum, } \\
\text { Large intestine, Liver, } \\
\text { Pancreas, Sigmoid, } \\
\text { Small Intestine, Stomach }\end{array}$ \\
\hline Endocrine system & $\begin{array}{l}\text { Subcutaneous adipose, } \\
\text { Thyroid }\end{array}$ & Adipose, Adrenal \\
\hline Exocrine system & $\begin{array}{l}\text { Skin, Breast mammary } \\
\text { tissue }\end{array}$ & \\
\hline Immune system & Spleen & $\begin{array}{l}\text { B cell (CD19+), B cell } \\
\text { (CD43-), MEP, Spleen }\end{array}$ \\
\hline Nervous system & $\begin{array}{l}\text { Cortex, Hippocampus, } \\
\text { Substantia nigra }\end{array}$ & Cerebellum, Cortex \\
\hline Renal system & Kidney & Bladder, Kidney \\
\hline Reproductive system & Ovary, Prostate, Testis & Ovary, Testis, Placenta \\
\hline Respiratory System & Lung & Lung \\
\hline Skeletal system & Skeletal muscle & \\
\hline
\end{tabular}

\section{Implementation}

As a wrapper for canonical RNA-Seq quantification algorithms, LocExpress takes full advantage of the locality of RNA-Seq data, and makes the abundance calls increasingly (Fig. 1, also refer to Additional file 3: Figure S2 for detailed workflow ).

The basic idea of LocExpress is straightforward: for a novel transcript, LocExpress will first infer its minimum spanning bundle (MSB), and make the (initial) expression call based on reads within the MSB only. Here, a bundle is defined as a continuous genomic region which is covered by overlapped reads (with no more than 50 bp gaps) as well as known gene models. The MSB is an independent unit in making an expression call (i.e. the expression estimation is independent in each bundle). And a novel transcript's MSB can be inferred by merging the transcript's spanning region with (overlapped) reference bundles which was derived by running StringTie [9] on the reference gene model (GENCODE v24 for human and vM9 for mouse, also see Additional file 1 for detailed RNA-Seq analysis procedure). After that, reads and existing gene models within the MSB are extracted and fed into the StringTie

Table 2 LocExpress supports expression estimation in common cell lines

\begin{tabular}{lll}
\hline & Human & Mouse \\
\hline Normal cell line & B cell (CD20+), GM12878, & 416B, C2C12, CH12, \\
& H1-hESC, HEK293, HMEC, & ES-E14, NIH-3 T3, Patski \\
& HUVEC, IMR90, & \\
& CD14+ monocytes & \\
Cancer cell line & A549, HeLa-S3, HepG2, & 10 T1/2, 416B, MEL \\
& K562, MCF-7, SK-N-SH_RA & \\
\hline
\end{tabular}

code. The resulting relative FPKM in each sample is further corrected for the fragment length by

$$
\text { Corrected FPKM }=\frac{\text { Relative FPKM } \times \text { Local total fragment length }}{\text { Global total fragment length }}
$$

Finally, these corrected FPKMs are geometrically normalized across samples, and reported to users, with replicates for the same cell type are averaged before.

All core modules are implemented in Python and Linux Shell, and the LocExpress website is developed based on Ruby on Rails (v4.2.6) with MySQL database supported. LocExpress is publicly available at http:// loc-express.cbi.pku.edu.cn.

\section{Results and discussion Performance evaluation}

To assess the performance of LocExpress, we simulated multiple user submissions by randomly choosing 300 transcripts from GENCODE reference gene models as "novel" transcripts per sample each time, resulting in 11,317 human transcripts and 9112 mouse transcripts in total. The evaluation was conducted on 40 human samples and 33 mouse samples independently. In each run, the chosen "novel" transcripts were removed from the original reference gene models and feed into the LocExpress one by one. Meanwhile, the output of StringTie ran in quantification-only mode (specified by “-e -B") with the full GENCODE gene models as reference annotation (specified by "-G") was taken as the "gold standard" for validating the correctness of LocExpress.

The evaluation shows that the LocExpress can correctly estimate abundance (Fig. 2a) within only seconds: the median time for a novel transcript per sample is $1.13 \mathrm{~s}$ in human and $0.43 \mathrm{~s}$ in mouse (Fig. 2b). While there are also dozen of extreme cases ( 9 in human and 17 in mouse) taking more than one minute per sample due to their complicated splicing structures as well as dense reads distribution in their MSB regions, the majority of transcripts takes several seconds only (90\% quantile of time cost per sample is $5.83 \mathrm{~s}$ in human and $2.83 \mathrm{~s}$ in mouse).

To further verify the performance of the LocExpress, we re-ran the evaluation on 3946 newly added transcripts in human GENCODE (v24 vs. v21), and 3874 in mouse GENCODE (vM9 vs. vM7, also see the "Evaluation on newly added transcripts" section in Additional file 1 for more details). Consistently, LocExpress is able to estimate expression abundance correctly for these novel transcripts (Additional file 1: Figure S1A) in nearly real-time (median time $0.71 \mathrm{~s}$ for human and $0.53 \mathrm{~s}$ for mouse, Additional file 1: Figure S1B).

\section{User interface}

LocExpress is designed to be intuitive. The most common operations (such as submitting transcript GTF 


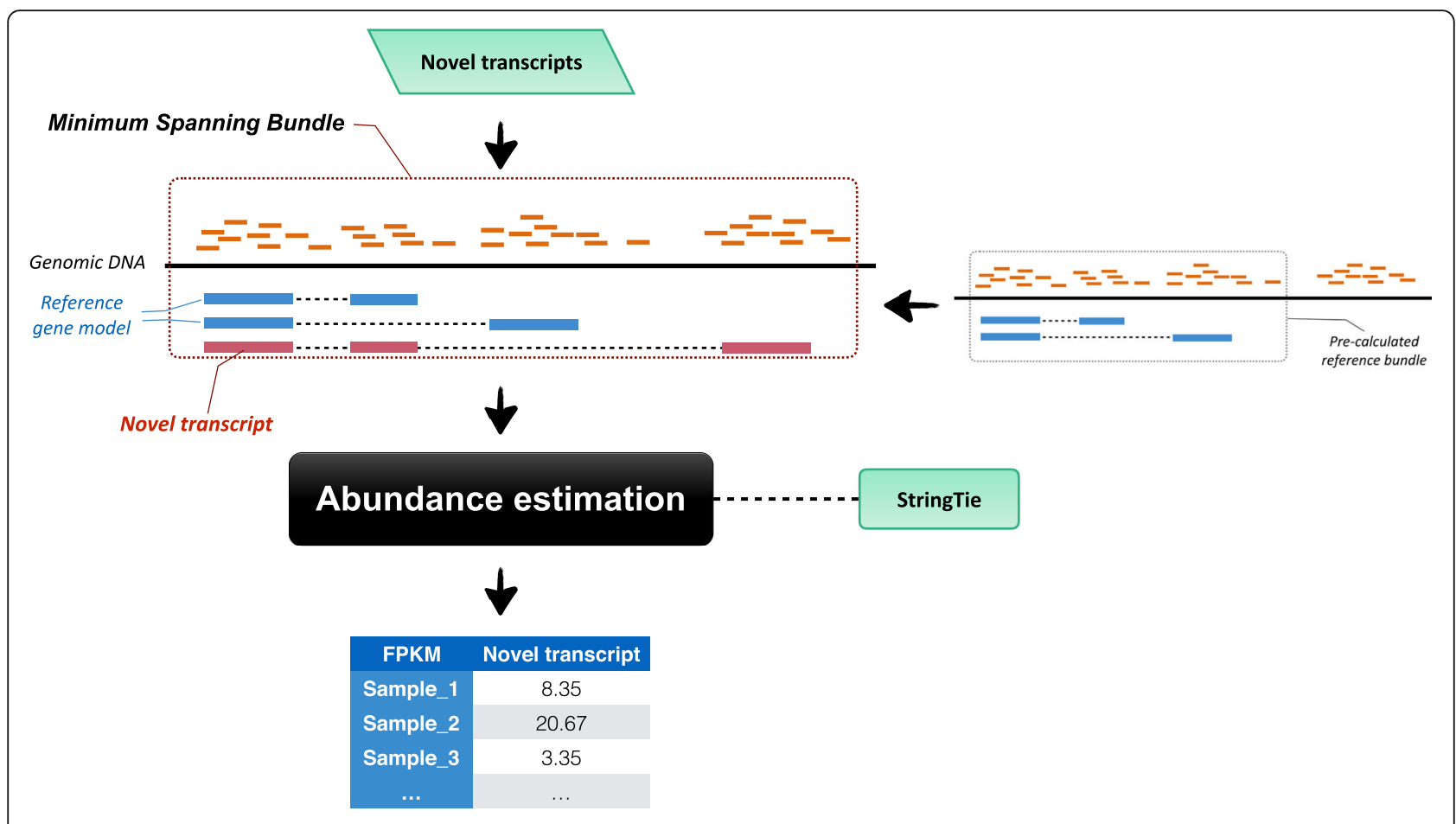

Fig. 1 The workflow of LocExpress. For an input novel transcript, LocExpress infers MSB for abundance estimation, and obtain FPKM accordingly

and checking results) can be performed with just a few clicks (Fig. 3). Users can select cell types and submit novel transcripts in GTF format at the "Home" page of LocExpress website (Fig. 3a). Then, users are led to the result page directly. Run status is showed on this page. If the job is not finished, this page will self-refresh every
$30 \mathrm{~s}$ (Fig. 3b). Users can keep the page open and wait the job to be finished, or just record the result URL and come back later. After the job is successfully finished, expression profiles of each transcript are rendered as bar plots (Fig. 3c). Users can also download the calculation results in text format as a ZIP (Fig. 3c).
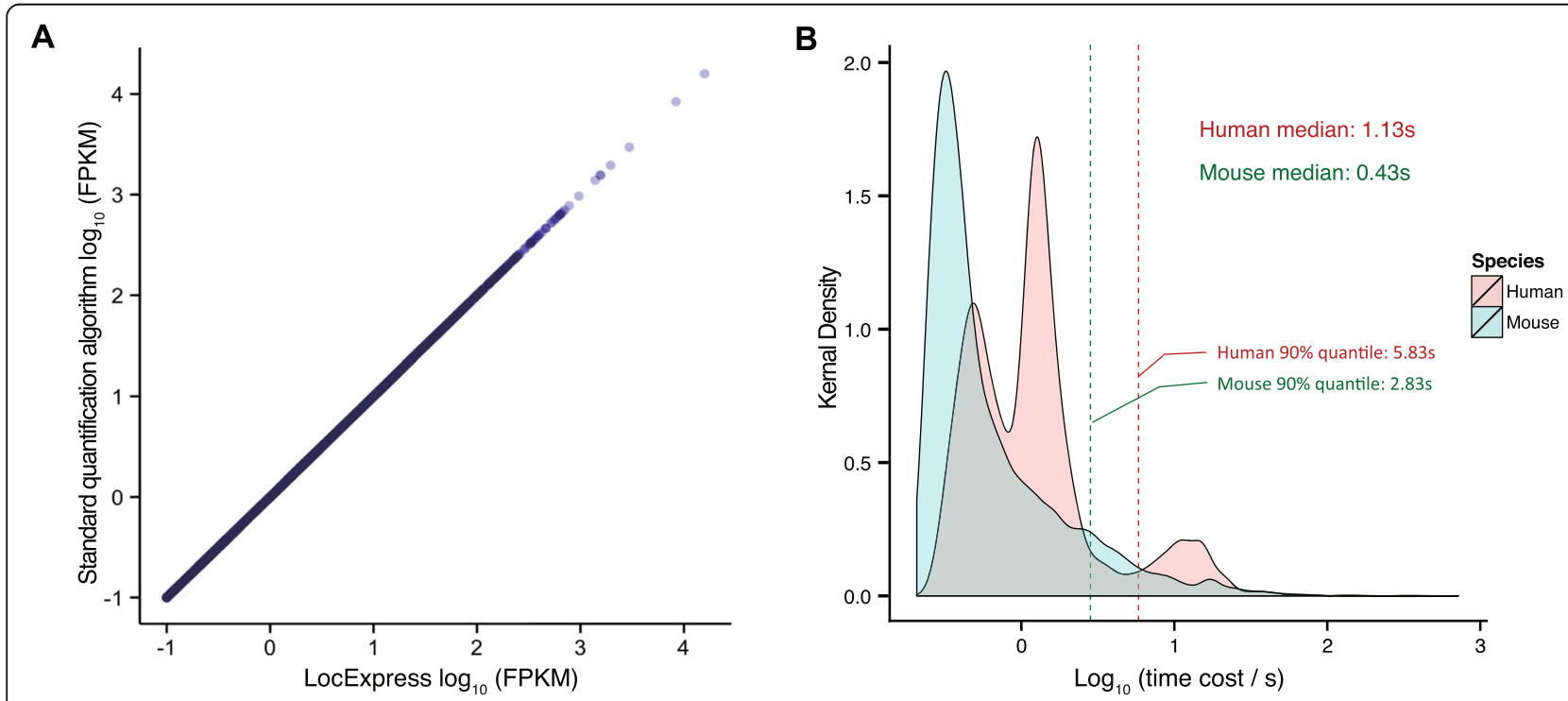

Fig. 2 Evaluation of LocExpress. a LocExpress arrives the same accuracy as the standard quantification algorithm. $\mathbf{b}$ The time cost per sample for a novel transcript. The median time cost is only $1.13 \mathrm{~s}$ in human and $0.43 \mathrm{~s}$ in mouse. The Linux box is configured with two Intel Xeon Processor E52670 v2 10C 2.5GHz CPUs, with 4*16GB ECC DDR3 $1866 \mathrm{MHz}$ memory 


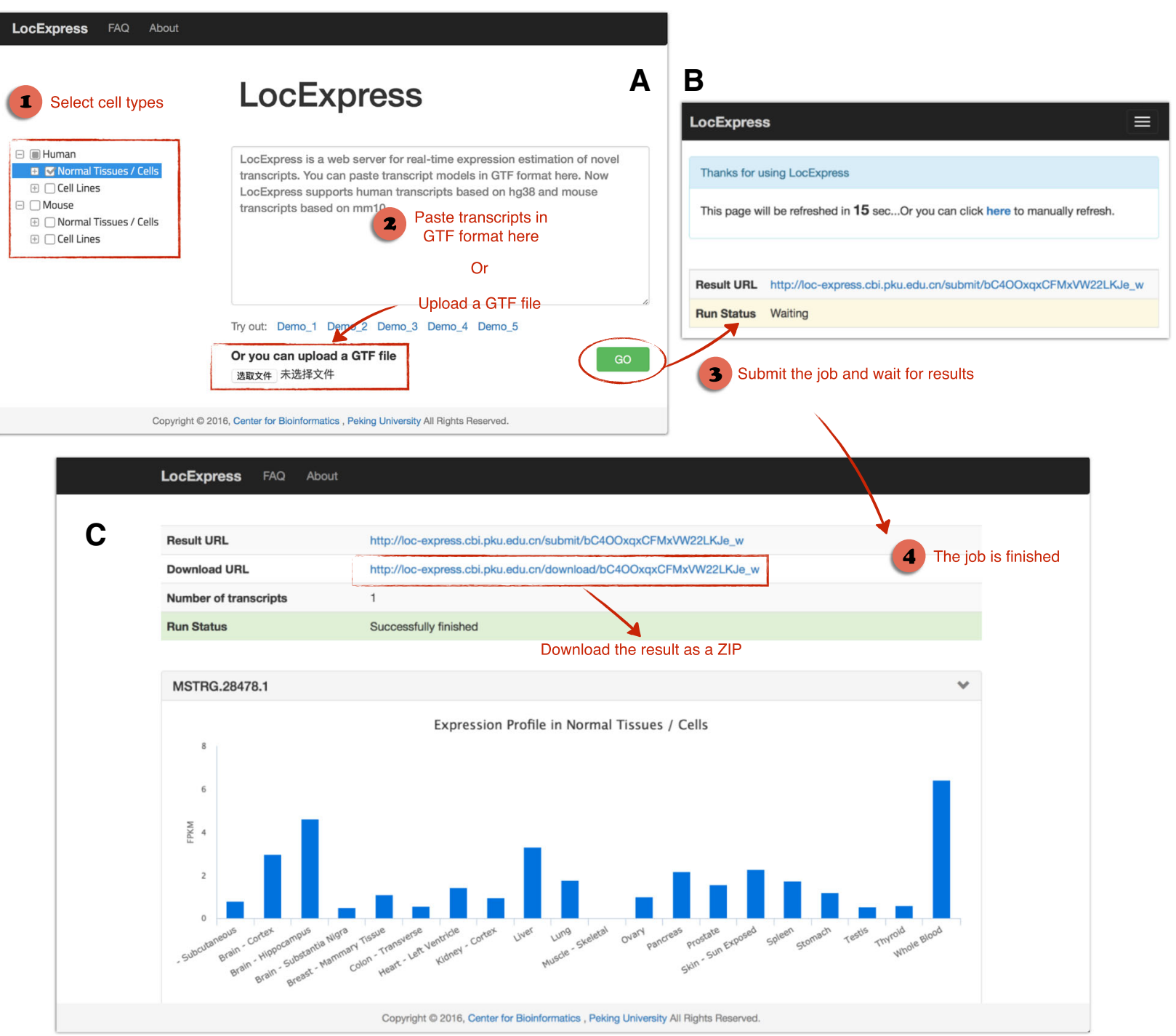

Fig. 3 The web interface of LocExpress. LocExpress web server is very convenient to use. a The "Home" page. Users can select cell types and submit transcript GTF in this page. b The "Run status" page which shows the running status of current submission. c The "Result" page. Expression profiles of submitted transcripts are rendered as bar charts

\section{Conclusions}

To the best of our knowledge, LocExpress is the only web server that can provide efficient real-time expression estimation for novel transcripts across multiple common human and mouse tissues and cell types. Taking advantage of RNA-Seq locality, LocExpress wraps canonical RNASeq quantification algorithm, archiving the same accuracy with overwhelming efficiency for novel transcripts. The median time cost is only about one second per submit per sample in human and half second in mouse. Powered by the intuitive web interface, LocExpress could be a useful tool for bench biologists to get the complete expression profile of their interested novel transcripts in just minutes with only a few clicks. In the future, we will continuously improve the LocExpress with more samples and more friendly interface based on users' feedback.

\section{Availability and requirements}

\section{Project name: LocExpress}

Project home page: http://loc-express.cbi.pku.edu.cn

Operating system: LocExpress can be accessed from any platform by using modern Web browsers (recommended but not limited to the latest version of Safari, Chrome and Firefox).

Programming languages: Python, Shell and Ruby

Any restrictions to use by non-academics: For nonacademic use, please contact loc-express@mail.cbi.pku.edu.cn. 


\section{Additional files}

Additional file 1: This file includes the supplementary table and figures, as well as additional methods and discussion. (DOCX $130 \mathrm{~kb}$ )

Additional file 2: This file lists human and mouse datasets used to build the LocExpress web server, including reads mapping information. (XLSX $18 \mathrm{~kb}$ )

Additional file 3: This file is Figure S2, a flowchart of detailed workflow of LocExpress. (PDF $332 \mathrm{~kb}$ )

\section{Abbreviations}

FPKM: Fragments per kilobase of exon per million fragments mapped; MSB: Minimum spanning bundle

\section{Acknowledgements}

Part of the analysis was performed on the Computing Platform of the Center for Life Sciences of Peking University. We thank for Ms. Christine Tiancheng Sun for her helpful comments during proofreading.

\section{Declarations}

This article has been published as part of BMC Genomics Volume 17 Supplement 13, 2016: 15th International Conference On Bioinformatics (INCOB 2016). The full contents of the supplement are available online at https://bmcgenet. biomedcentral.com/articles/supplements/volume-17-supplement-13.

\section{Funding}

This work was supported by funds from the China 863 Program (2015AA020108), the Seeding Grant for Medicine and Life Sciences of Peking University (2014-MB-13), the International Collaboration Program for Proteome Biological Big Data and Standard System (2014DFB30030), as well as the State Key Laboratory of Protein and Plant Gene Research. The research of G.G. was supported in part by the National Program for Support of Top-notch Young Professionals. Publication charges for this article have been funded by the State Key Laboratory of Protein and Plant Gene Research.

\section{Availability of data and materials}

Not applicable.

\section{Authors' contributions}

GG conceived this work. MH implemented the local strategy and built the web server. FT helped most of the RNA-Seq analysis and evaluation. SJ helped with novel transcript model. LK helped with the infrastructure of web server. DCY helped with some web pages. GG and $\mathrm{MH}$ wrote the manuscript. All authors read and approved the final manuscript.

\section{Competing interests}

The authors declare that they have no competing interests.

\section{Consent for publication}

Not applicable.

\section{Ethics approval and consent to participate}

Not applicable.

\section{Author details}

${ }^{1}$ State Key Laboratory of Protein and Plant Gene Research, College of Life Sciences, Center for Bioinformatics, Peking University, Beijing 100871, People's Republic of China. ${ }^{2}$ Peking-Tsinghua Center for Life Sciences, Academy for Advanced Interdisciplinary Studies, Peking University, Beijing 100871, People's Republic of China.

Published: 22 December 2016

\section{References}

1. Harrow J, Frankish A, Gonzalez JM, Tapanari E, Diekhans M, Kokocinski F, Aken BL, Barrell D, Zadissa A, Searle S, et al. GENCODE: the reference human genome annotation for The ENCODE Project. Genome Res. 2012;22(9):1760-74.

2. Guttman M, Amit I, Garber M, French C, Lin MF, Feldser D, Huarte M, Zuk O, Carey BW, Cassady JP, et al. Chromatin signature reveals over a thousand highly conserved large non-coding RNAs in mammals. Nature. 2009; 458(7235):223-7.

3. Petryszak R, Keays M, Tang YA, Fonseca NA, Barrera E, Burdett T, Fullgrabe A, Fuentes AM, Jupp S, Koskinen S, et al. Expression Atlas update-an integrated database of gene and protein expression in humans, animals and plants. Nucleic Acids Res. 2016;44(D1):D746-52.

4. Wu C, Orozco C, Boyer J, Leglise M, Goodale J, Batalov S, Hodge CL, Haase J, Janes J, Huss 3rd JW, et al. BioGPS: an extensible and customizable portal for querying and organizing gene annotation resources. Genome Biol. 2009; 10(11):R130.

5. Chalifa-Caspi V, Shmueli O, Benjamin-Rodrig H, Rosen N, Shmoish M, Yanai I, Ophir R, Kats P, Safran M, Lancet D. GeneAnnot: interfacing GeneCards with high-throughput gene expression compendia. Brief Bioinform. 2003;4(4): 349-60.

6. D'Antonio M, D'Onorio De Meo P, Pallocca M, Picardi E, D'Erchia AM, Calogero RA, Castrignano T, Pesole G. RAP: RNA-Seq Analysis Pipeline, a new cloud-based NGS web application. BMC Genomics. 2015;16:S3.

7. Kalari KR, Nair AA, Bhavsar JD, O'Brien DR, Davila JI, Bockol MA, Nie J, Tang X, Baheti S, Doughty JB, et al. MAP-RSeq: Mayo Analysis Pipeline for RNA sequencing. BMC Bioinformatics. 2014;15:224.

8. Wang Y, Mehta G, Mayani R, Lu J, Souaiaia T, Chen Y, Clark A, Yoon HJ, Wan L, Evgrafov OV, et al. RsegFlow: workflows for RNA-Seq data analysis. Bioinformatics. 2011;27(18):2598-600

9. Pertea M, Pertea GM, Antonescu CM, Chang TC, Mendell JT, Salzberg SL. StringTie enables improved reconstruction of a transcriptome from RNA-seq reads. Nat Biotechnol. 2015;33(3):290-5.

\section{Submit your next manuscript to BioMed Central and we will help you at every step:}

- We accept pre-submission inquiries

- Our selector tool helps you to find the most relevant journal

- We provide round the clock customer support

- Convenient online submission

- Thorough peer review

- Inclusion in PubMed and all major indexing services

- Maximum visibility for your research

Submit your manuscript at www.biomedcentral.com/submit 